\title{
Kimura's Disease of the Orbit Successfully Treated with Radiotherapy Alone: A Case Report
}

\author{
Yoshio Monzen $^{a} \quad K^{2}$ atsuzo Kiya ${ }^{b} \quad$ Takashi Nishisaka $^{c}$ \\ Departments of ${ }^{a}$ Radiology, ${ }^{b}$ Neurosurgery and ${ }^{c}$ Pathology, \\ Hiroshima Prefectural Hospital, Hiroshima, Japan
}

\section{Key Words}

Kimura's disease $\cdot$ Orbit $\cdot$ Radiotherapy

\begin{abstract}
We performed radiotherapy in a 28-year-old patient with Kimura's disease of the orbit. Irradiation with $21.6 \mathrm{~Gy}$ was administered to the tumor bed with a single dose of $1.8 \mathrm{~Gy}$ in 5 weekly fractions delivered via a high-energy linear accelerator (6-MV X-ray). Complete remission of the tumor and improvements in the neurological findings were achieved. Neither tumor regrowth nor late complications were detected up to 84 months after radiotherapy. Radiotherapy is an effective treatment for Kimura's disease of the orbit.
\end{abstract}

(C) 2014 S. Karger AG, Basel

\section{Introduction}

Kimura's disease of the orbit is a rare chronic inflammatory disorder affecting the skin and subcutaneous tissue. We herein report a patient with Kimura's disease of the orbit that was successfully treated with radiotherapy alone.

\section{Case Report}

A 28-year-old male presented to the Department of Ophthalmology in February 2006 with a history of blepharoptosis, exophthalmos, a reduction of visual acuity as well as a visual field defect in the right eye. The eyesight of the right eye was 0.07 and that of the left eye was 1.2. Inferior hemianopsia with a scotoma in the center was shown by the Goldmann 
Monzen et al.: Kimura's Disease of the Orbit Successfully Treated with Radiotherapy Alone: A Case Report

perimetry. A relative afferent pupillary defect (RAPD) in the right eye was detected. Double vision and disturbance of the lateral gaze in the right eye were also pointed out. A mass on the right side of the neck had been observed 19 years earlier and the patient had been pathologically diagnosed as having Kimura's disease. The diagnosis of a tumor of the right orbit was made based on clinical and MRI findings (fig. 1).

Blood tests showed peripheral blood eosinophilia (15\%; normal 6\%) and elevated serum immunoglobulin E (IgE: 4,470 IU $/ \mathrm{ml}$; normal $170 \mathrm{IU} / \mathrm{ml}$ ). The total leukocyte count was $6,300 / \mathrm{mm}^{3}$. A differential white blood cell count performed at that time showed $56 \%$ neutrophils, $23 \%$ lymphocytes, $15 \%$ eosinophils and $6 \%$ monocytes.

A biopsy of the mass of the right orbit was performed by a neurosurgeon. A histological examination of the hematoxylin- and eosin-stained sections of the resected mass revealed a proliferation of the fibrous connective tissue and small vessels with an infiltration of the lymphocytes and eosinophils. There was no evidence of lymphoid follicle growth or malignancy, leading to a pathological diagnosis of Kimura's disease after considering the blood test results (fig. 2).

A neurosurgeon highly recommended radiotherapy and consulted a radiation oncologist. Radiation therapy was started in May 2006. Irradiation with 21.6 Gy was administered to the tumor bed in a single dose of 1.8 Gy in 5 weekly fractions via a high-energy linear accelerator (6-MV X-ray). A single anterior oblique portal was used to avoid radiation exposure of the right lens (fig. 3). Lens protection was not used during radiotherapy. Eye drops for radiation-induced conjunctivitis and an ointment to treat radiation-induced dermatitis were also not prescribed. The tumor gradually diminished and ultimately disappeared. Four months after the completion of radiotherapy, the eyesight of the right eye was 1.5, which was the same as that of the left eye, and an improvement of the inferior hemianopsia as well as the disappearance of the scotoma in the center were demonstrated by the Goldmann perimetry. Improvements in the exophthalmos, double vision and a RAPD of the right eye were achieved. The irradiated right eye ball did not develop dry eye syndrome or a cataract. There was no evidence of radiation-induced dermatitis or radiation neuropathy. Neither tumor regrowth nor late complications were detected up to 84 months after radiotherapy (fig. 4). Both the elevated serum IgE level and eosinophilia gradually decreased. The serum IgE level was $470 \mathrm{IU} / \mathrm{ml} 84$ months after radiotherapy and the peripheral eosinophil level was 7.9\% in June 2013.

\section{Discussion}

Kimura's disease, often called eosinophilic granuloma of the soft tissue, is a benign chronic granulomatous inflammatory disorder of unknown etiology [1-3]. As peripheral blood eosinophilia with an elevated serum IgE level is a common finding, the involvement of an allergic reaction or autoimmune disease has been suggested.

The disease is endemic in Asia (especially Japan and Southeast Asia), and the incidence rate is high in the first and third decades of life. Common primary sites of involvement include the parotid regions followed by the lacrimal glands, the submandibular regions, the neck, the axillary regions, the elbows and the inguinal region. Orbital involvement in Kimura's disease is infrequent; however, when it occurs, it usually affects the eyelids or the lacrimal glands [4].

The clinical presentation consists of subcutaneous nodules or superficial lymphadenopathy. Complications of the disease include nephritis, asthma and urticaria. 
Monzen et al.: Kimura's Disease of the Orbit Successfully Treated with Radiotherapy Alone: A Case Report

No standard treatment for Kimura's disease has been established. Steroid therapy induces a marked reduction in tumor size. However, the tumor often increases again as the patient is weaned from systemic steroids. It has been reported that a partial excision is useful for the following reasons: (a) the tumor is controlled without complete resection and (b) Kimura's disease has not been reported to exhibit malignant transformation [5]. Radiotherapy is effective against Kimura's disease [5, 6]. The efficacy of radiotherapy is influenced by the quantity of connective tissue and fibrous components inside the lesion.

The optimal dose sufficient to achieve local control is approximately 26-30 Gy, regardless of the tumor size [5]. Okazaki [7] reported that drug therapy is the first choice in young patients with Kimura's disease, and, if drug therapy is ineffective, complete resection is recommended. If performing complete resection is difficult because the tumor is infiltrative, radiotherapy ( $20 \mathrm{~Gy}$ ) is prescribed for a lesion. Drug therapy is the first choice in middleaged or elderly patients with Kimura's disease. If drug therapy is ineffective, radiotherapy (30 Gy) is administered.

We believe that the treatment strategy for Kimura's disease of the orbit should follow the treatment plan for Kimura's disease described above. Therapy for Kimura's disease of the orbit and the ocular adnexa includes observation due to the potential for a spontaneous regression, although excision, steroid therapy and radiotherapy have been applied in various combinations $[8,9]$. In patients with Kimura's disease of the orbit treated with steroid therapy or a complete resection, local control ranges from 7 months to 4 years [9-11].

There have been very few reports of the use of radiotherapy for Kimura's disease of the orbit. In a previous case, Kimura's disease of the orbit was treated with radiotherapy (28 Gy) alone. Although local control was obtained, a local relapse was observed within 3 years [8].

Kimura's disease of the orbit was treated with radiotherapy (21.6 Gy) alone in the present case. The decrease in visual acuity and the visual field defect were considered to have been caused by the dysfunction of the right optic nerve which was involved by the tumor. We thought that a tumor reduction could bring an improvement in the visual acuity and reduce visual field loss. Complete remission of the tumor and improvements in the neurological findings were obtained for 84 months after radiotherapy. Although providing a long-term follow-up period is essential, radiotherapy is an effective treatment for Kimura's disease of the orbit.

\section{Disclosure Statement}

The authors declare no conflicts of interest.

\section{References}

1 Larroche C, Blétry O: Kimura's disease. Orphanet Encyclopedia 2005. https://www.orpha.net/data/patho/GB/uk-kimura.pdf.

2 Chitapanarux I, Ya-in C, Kittichest R, et al: Radiotherapy in Kimura's disease: a report of eight cases. J Med Assoc Thai 2007;90:1001-1005.

3 Kilciksiz S, Calli C, Eski E, Topcugil F, Bener S: Radiotherapy for Kimura's disease: case report and review of the literature. J BUON 2007;12:277-280.

-4 Goncalves AC, Moritz RB, Aldred VL, Monteiro ML: Bilateral extraocular muscles enlargement from Kimura's disease of the orbit. Indian J Ophthalmol 2013, Epub ahead of print.

5 Hareyama M, Oouchi A, Nagakura H, et al: Radiotherapy for Kimura's disease: the optimum dose. Int J Radiat Oncol Biol Phys 1998;40:647-651.

-6 Chang AR, Kim K, Kim HJ, Kim IH, Park CI, Jun YK: Outcomes of Kimura's disease after radiotherapy or nonradiotherapeutic treatment modalities. Int J Radiat Oncol Biol Phys 2006;65:1233-1239. 
Case Reports in

Ophthalmology

\begin{tabular}{l|l}
\hline Case Rep Ophthalmol 2014;5:87-91 \\
\hline DOI: 10.1159/000360789 & $\begin{array}{l}\text { ○ 2014 S. Karger AG, Basel } \\
\text { www.karger.com/cop }\end{array}$ \\
\hline
\end{tabular}

Monzen et al.: Kimura's Disease of the Orbit Successfully Treated with Radiotherapy Alone: A Case Report

7 Okazaki A: Kimura's disease. Japanese Journal of Clinical Radiology 2002;47:144-154.

$>8$ Buggage RR, Spraul CW, Wojno TH, Grossniklaus HE: Kimura disease of the orbit and ocular adnexa. Surv Ophthalmol 1999;44:79-91.

-9 Yeung EY, Ma L: Bilateral orbital Kimura's disease in a young Asian man. Chang Gung Med J 2002;25:45-49.

10 Ko KF: Kimura's disease of the orbit. J Hong Kong Med Assoc 1990;42:227-228.

11 Kanazawa S, Gong H, Kitaoka T, Amemiya T: Eosinophilic granuloma (Kimura's disease) of the orbit: a case report. Graefe's Arch Clin Exp Ophthalmol 1999;237:518-521.

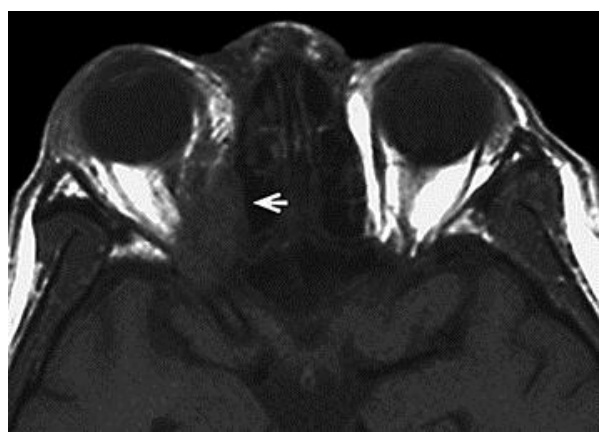

Fig. 1. Axial T1-weighted spin-echo imaging (491/11) (TR/TE) performed before radiotherapy demonstrated an isointense tumor in the right orbital space and the right ethmoid sinus (arrow). The right optic nerve was involved by the tumor.

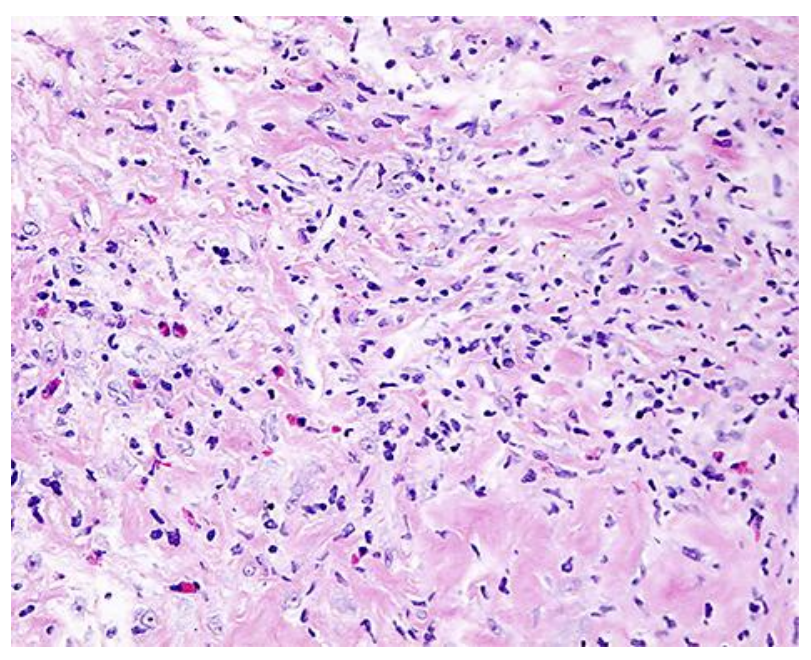

Fig. 2. Histological section showing Kimura' s disease. HE. 
Case Reports in

Ophthalmology
Case Rep Ophthalmol 2014;5:87-91

DOI: $10.1159 / 000360789$

Monzen et al.: Kimura's Disease of the Orbit Successfully Treated with Radiotherapy Alone: A Case Report

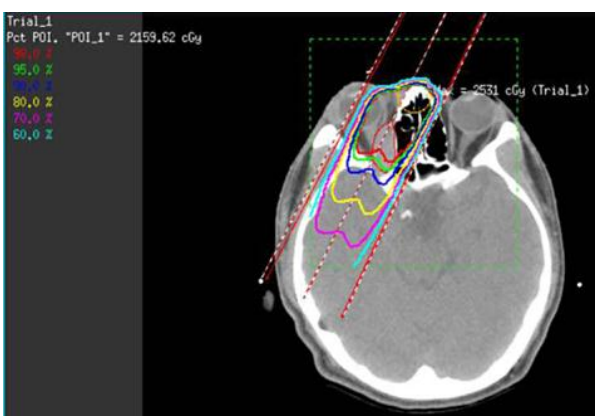

Fig. 3. Dose distribution. One oblique field is shown.

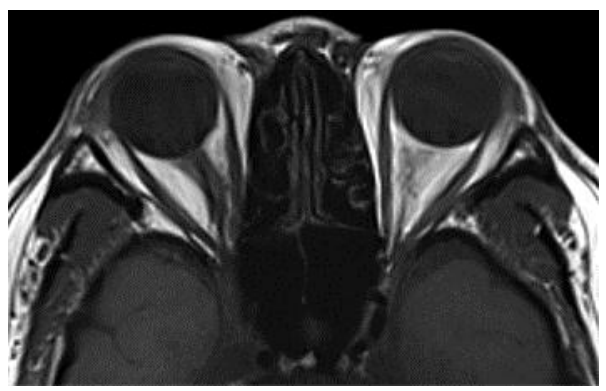

Fig. 4. Eighty-four months after radiotherapy, axial T1-weighted spin-echo imaging (440/150) (TR/TE) demonstrated the disappearance of the right orbital tumor. 